\title{
NMR- and MS-based untargeted metabolomic study of stool and serum samples from patients with anorexia nervosa
}

Petra Tomášováa,, , Petra Procházkovác, Radka Roubalová c, Jiří Dvořák c, Helena Tlaskalová-

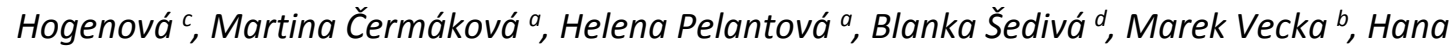
Papežová e, Marek Kuzma ${ }^{a}$

${ }^{a}$ Laboratory of Molecular Structure Characterization, Institute of Microbiology, Academy of Sciences of the Czech Republic, v.v.i., Vídeňská 1083, 14220 Prague 4, Czech Republic

$\mathrm{b} 4^{\text {th }}$ Medical Department, First Faculty of Medicine, Charles University and General Faculty Hospital in Prague, U Nemocnice 2, 12108 Praha 2, Czech Republic

c Laboratory of Cellular and Molecular Immunology, Institute of Microbiology, Academy of Sciences of the Czech Republic, v.v.i., Vídeňská 1083, 14220 Prague 4, Czech Republic

${ }^{d}$ Faculty of Applied Sciences, University of West Bohemia, Univerzitní 8, 30614 Plzeň, Czech Republic eDepartment of Psychiatry, First Faculty of Medicine, Charles University and General University Hospital in Prague, Ke Karlovu 11, 12108 Prague 2, Czech Republic

\begin{tabular}{|c|c|c|}
\hline Item & Title & Page \\
\hline Fig. S1 & MS-based metabolomics of serum & $\mathrm{S}-2$ \\
\hline Fig. S2 & MS-based metabolomics of stool & S-3 \\
\hline Fig. S3 & NMR-based metabolomics of serum & S-4 \\
\hline Fig. S4 & NMR-based metabolomics of stool & S-4 \\
\hline Table S1 & Partial correlation of biochemical parameters with metabolites levels in serum of all samples & S-5 \\
\hline Table S2 & $\begin{array}{c}\text { Correlation of serum metabolites levels with biochemical and anthropometric parameters in patients with } \\
\text { anorexia nervosa group after treatment }\end{array}$ & S-5 \\
\hline
\end{tabular}


Scores Plot

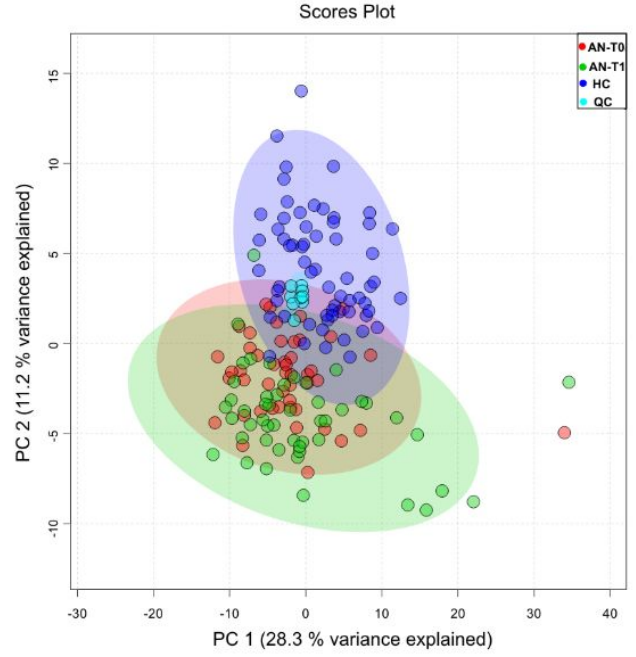

A

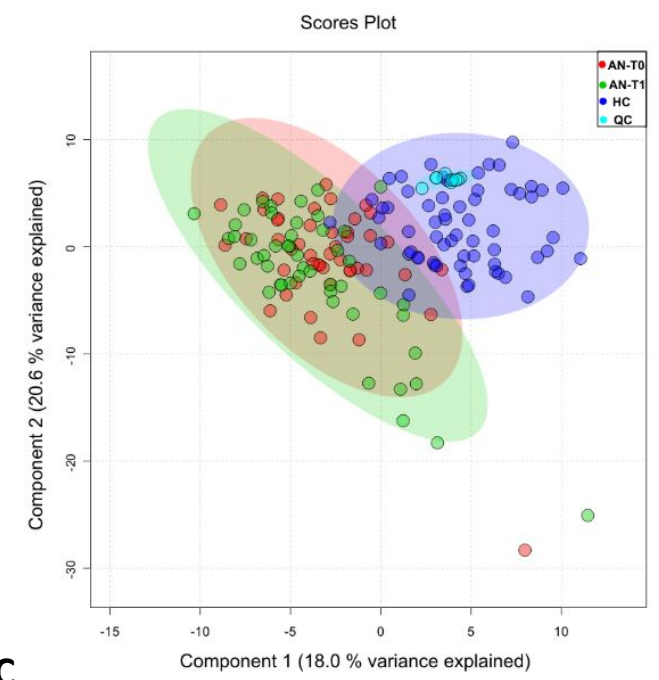

Scores Plo

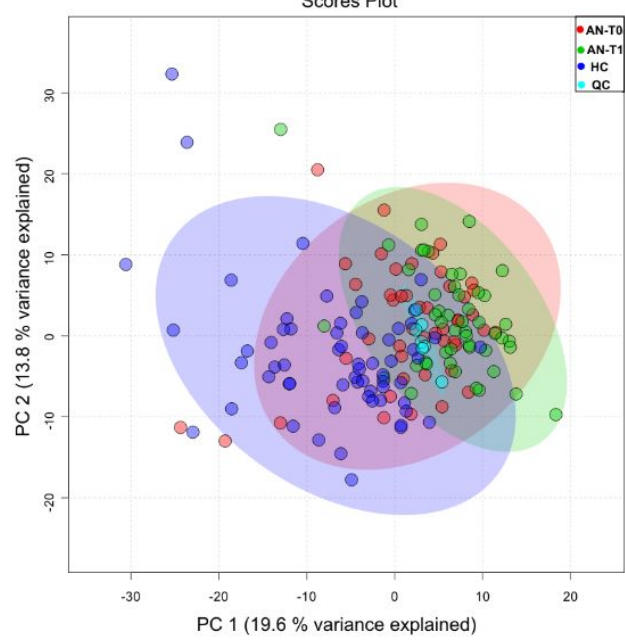

B

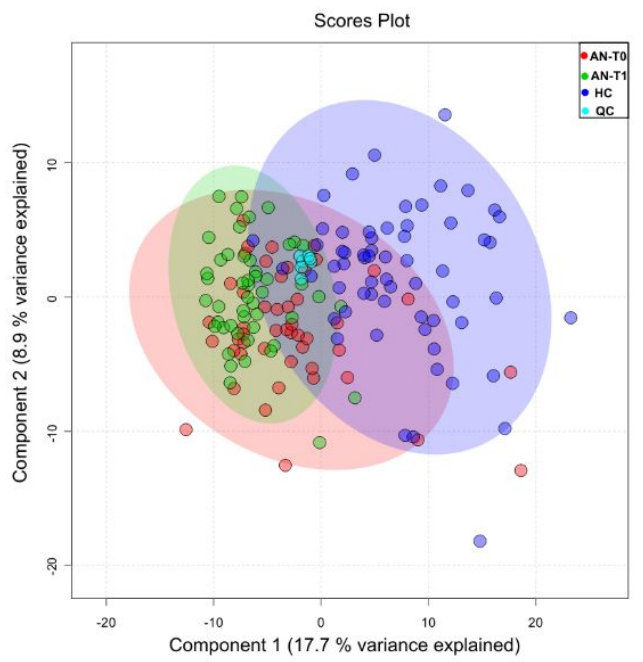

Fig. S1 MS-based metabolomics of serum. A) PCA score plot, positive ionization mode; B) PCA score plot, negative ionization mode; C) PLS-DA score plot, positive ionization mode; the results of leave-one-out cross validation: number of components: 2, accuracy: 0.655, $\mathrm{R}^{2}=0.532, \mathrm{Q}^{2}=0.470$, $\mathrm{p}$ value of permutation test with 2000 repetitions $\left.<0.001 ; \mathrm{D}\right) \mathrm{PLS}-\mathrm{DA}$ score plot, negative ionization mode; the results of leave-one-out cross validation: number of components: 2 , accuracy: $0.669, \mathrm{R}^{2}=0.357, \mathrm{Q}^{2}=0.215, \mathrm{p}$ value of permutation test with 2000 repetitions <0.001; color coding: patients with anorexia nervosa at hospital admission AN-T0 - red points, patients with anorexia nervosa at hospital release AN-T1- green points, healthy controls HC - blue points, quality control samples QC - turquoise points. 


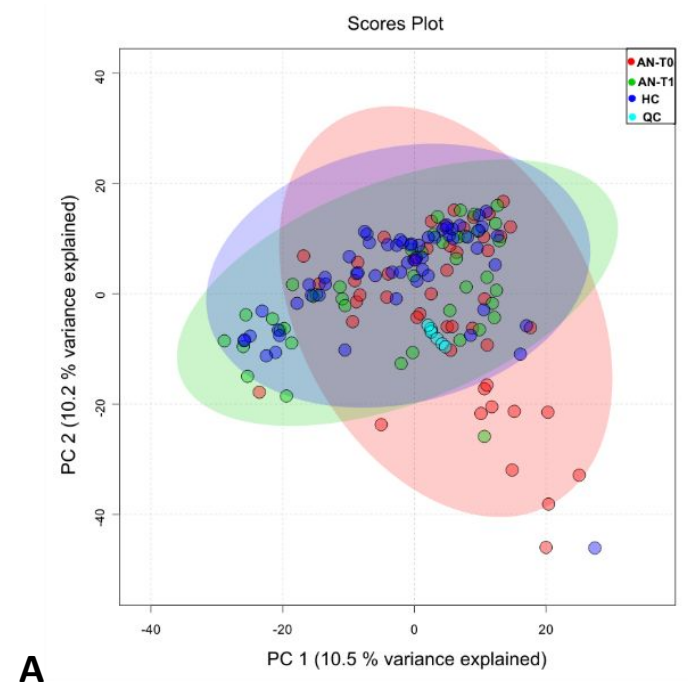

A

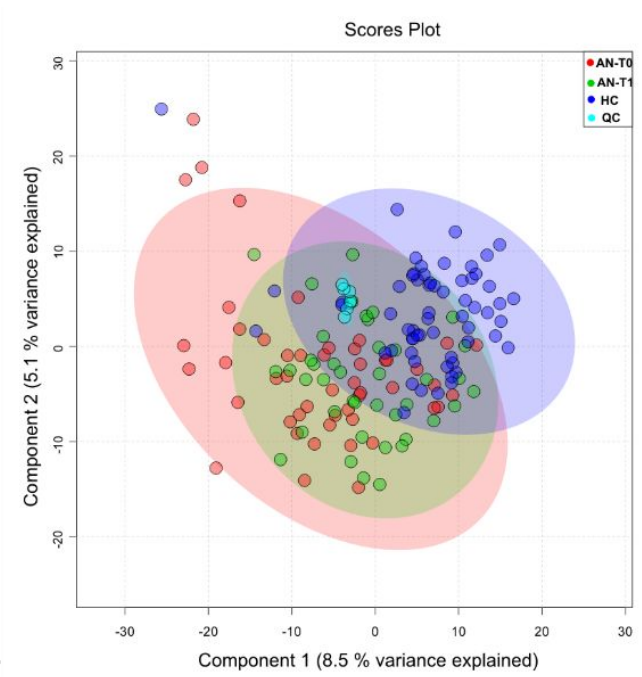

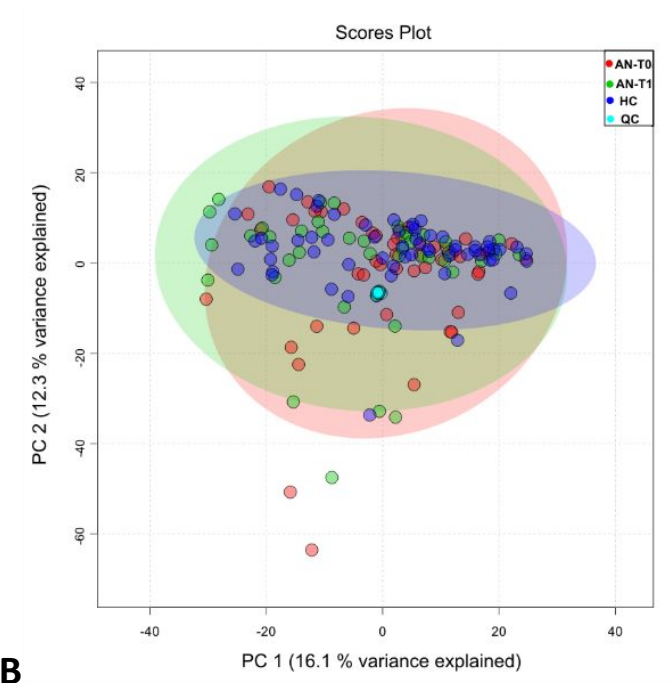

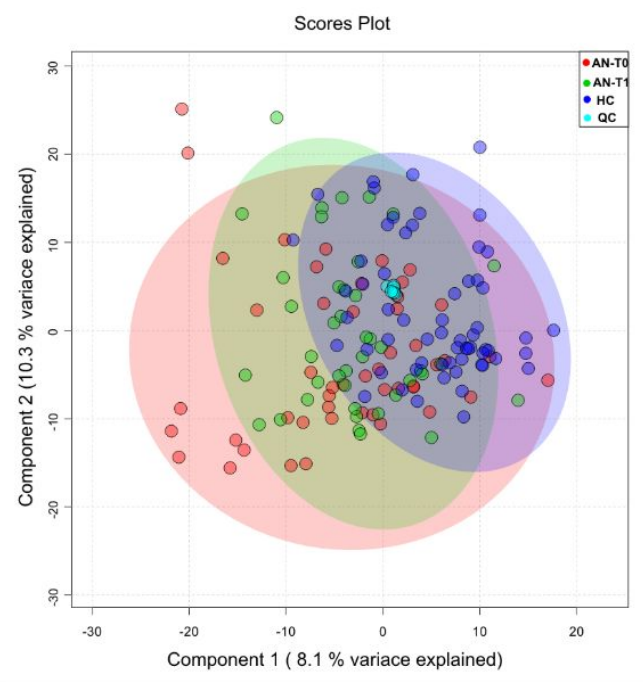

Fig. S2 MS-based metabolomics of stool. A) PCA score plot, positive ionization mode; B) PCA score plot, negative ionization mode; C) PLS-DA score plot, positive ionization mode; the results of leave-one-out cross validation: number of components: 2, accuracy: 0.519, $\mathrm{R}^{2}=0.405, \mathrm{Q}^{2}=0.132, \mathrm{p}$ value of permutation test with 2000 repetitions $\left.0.002 ; \mathrm{D}\right) \mathrm{PLS}-\mathrm{DA}$ score plot, negative ionization mode; the results of leave-one-out cross validation: number of components: 2 , accuracy: $0.453, R^{2}=0.292, Q^{2}=0.114, p$ value of permutation test with 2000 repetitions 0.001; color coding: patients with anorexia nervosa at hospital admission AN-TO red points, patients with anorexia nervosa at hospital release AN-T1- green points, healthy controls HC - blue points, quality control samples QC - turquoise points. 

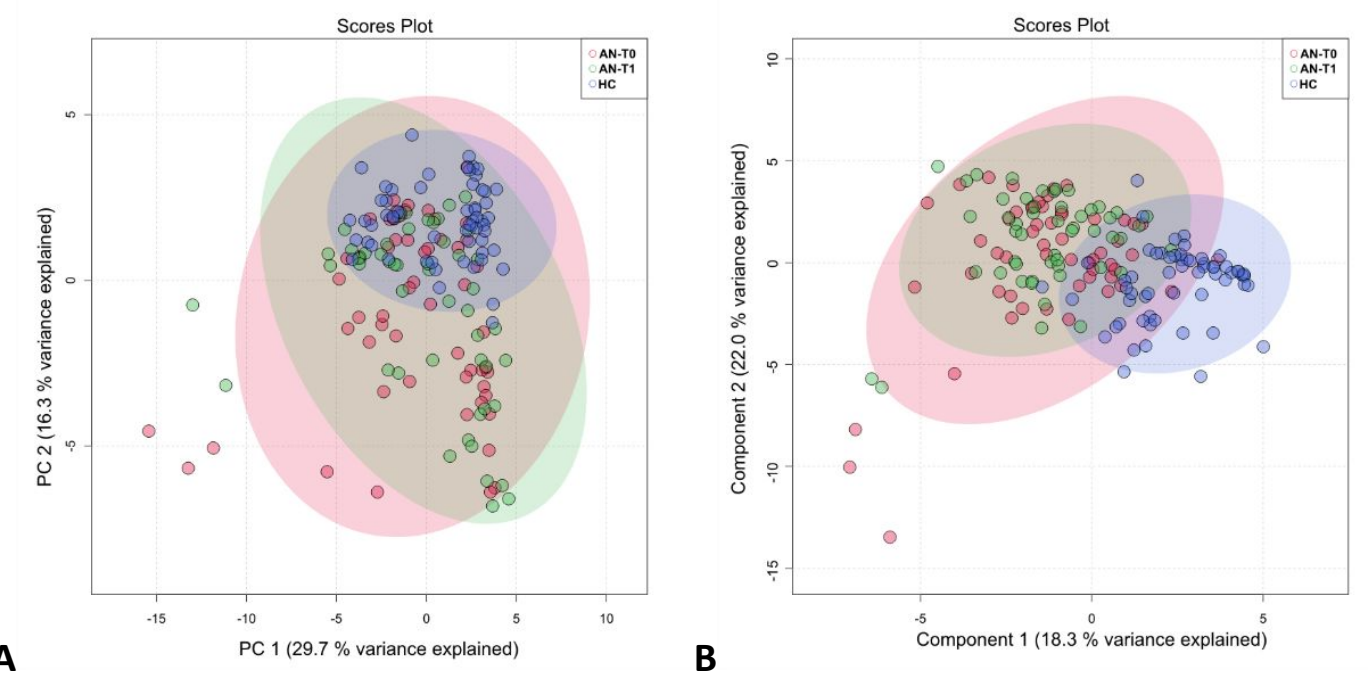

Fig. S3 NMR-based metabolomics of serum. A) PCA score plot; B) PLS-DA score plot; the results of leave-one-out cross validation: number of components: 2, accuracy: 0.591, $\mathrm{R}^{2}=0.148, \mathrm{Q}^{2}=0.114, \mathrm{p}$ value of permutation test with 2000 repetitions $<0.001$; color coding: patients with anorexia nervosa at hospital admission AN-TO - red points, patients with anorexia nervosa at hospital release AN-T1- green points, healthy controls HC - blue points.
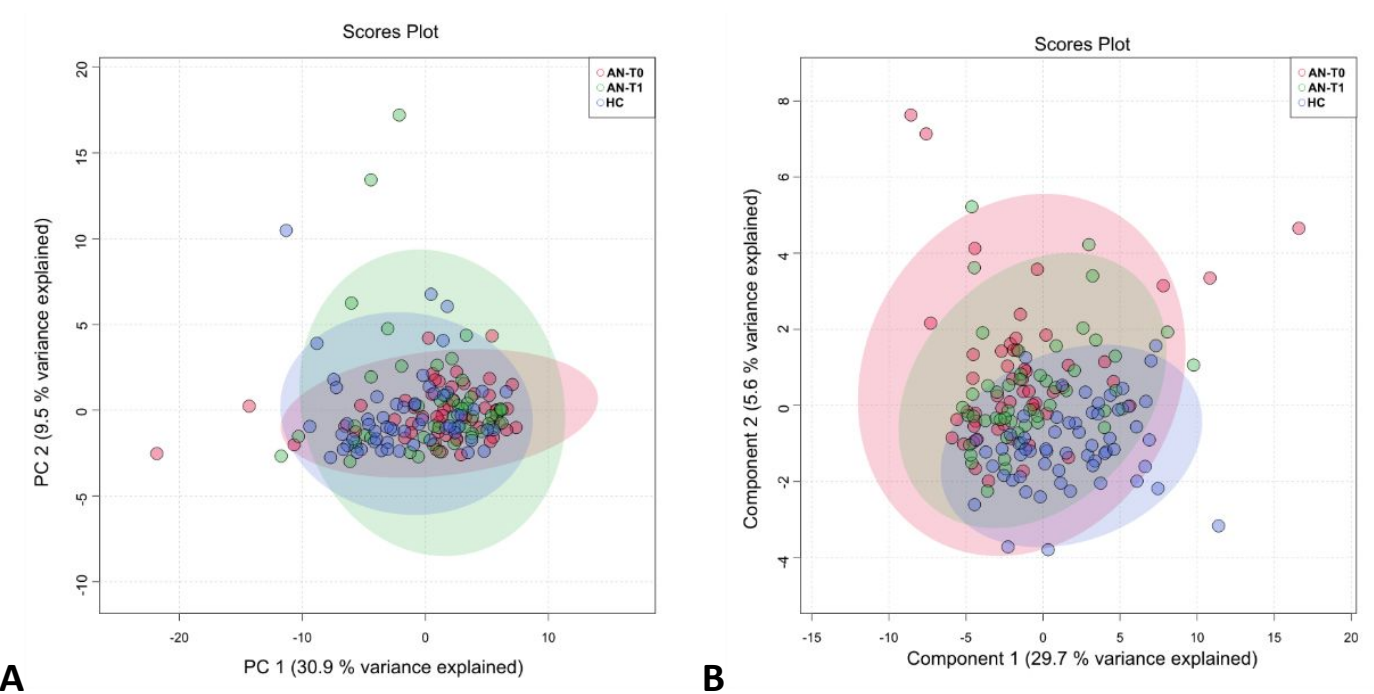

Fig. S4 NMR-based metabolomics of stool. A) PCA score plot; B) PLS-DA score plot; the results of leave-one-out cross validation: number of components: 2 , accuracy: $0.511, R^{2}=0.165$, $Q^{2}=0.035$, $p$ value of permutation test with 2000 repetitions $<0.001$; color coding: patients with anorexia nervosa at hospital admission AN-TO - red points, patients with anorexia nervosa at hospital release AN-T1- green points, healthy controls HC - blue points. 
Table S1 Partial correlation of biochemical parameters with metabolites levels in serum of all samples

\begin{tabular}{|l|lllll|}
\hline Metabolite & TAG & BMI & Body fat mass & fT4 & Albumin \\
\hline Lactate & $0.311^{* * *}$ & - & - & - & - \\
Pyruvate & $0.375 * * *$ & - & - & - & - \\
Isoleucine & - & - & - & - & $0.450 * * *$ \\
Oxoisocaproate & - & - & - & - & $0.448 * * *$ \\
PC(30:0) & - & - & - & $0.304 * * *$ & - \\
PC(32:1) & $0.478 * * *$ & - & - & - & - \\
PC(36:4) & - & $0.342 * * *$ & - & - & - \\
PC(38:4) & - & - & $0.354 * * *$ & - & - \\
Acetylcarnitine & - & - & - & $0.348 * * *$ & - \\
\hline
\end{tabular}

Data are presented as R values; ${ }^{*} p<0.05, * * p<0.01, * * * p<0.001$; TAG- triacylglycerols, fT4- free thyroxin

Table S2 Correlation of serum metabolites levels with biochemical and anthropometric parameters in patients with anorexia nervosa group after treatment

\begin{tabular}{|l|lllllll|}
\hline Metabolite & TAG & BMI & Body fat mass & fT4 & TSH & IL-6 & TNF- $\alpha$ Albumin \\
\hline Oxoisocaproate & - & - & - & - & - & - & - \\
Choline & - & - & - & - & - & - & $-0.312^{*}$ \\
LysoPE (16:0) & $0.452^{* *}$ & - & - & - & - & - & - \\
PC(30:0) & $0.310^{*}$ & - & - & - & - & - & - \\
PC(32:1) & $0.620^{* * *}$ & - & - & - & - & - & - \\
Carnitine & - & - & - & $0.404^{* *}$ & - & - & - \\
Acetylcarnitine & - & - & - & $0.489 * * *$ & - & - & - \\
Hexanoylcarnitine & - & $0.333^{*}$ & $0.505^{* * *}$ & - & - & $0.321^{*}$ & - \\
Hexenoylcarnitine & - & - & $0.335^{*}$ & - & - & - & - \\
Butyrylcarnitine & - & - & - & - & $0.312^{*}$ & - & - \\
\hline
\end{tabular}

Data are presented as R values; ${ }^{*} p<0.05, * * p<0.01, * * * p<0.001$; TAG- triacylglycerols, TSH- thyroid-stimulating hormone, fT4- free

thyroxin, IL-6- interleukin-6, TNF- $\alpha$ - tumor necrosis factor- $\alpha$ 
Table S3 Correlation of serum metabolites levels with biochemical and anthropometric parameters in control group

\begin{tabular}{|l|lllll|}
\hline Metabolite & TAG & BMI & Body fat mass & TNF- $\alpha$ & Albumin \\
\hline Isoleucine & - & - & - & - & $0.428^{* * *}$ \\
Oxoisocaproate & - & - & - & - & $0.400^{* *}$ \\
Choline & - & - & - & - & $-0.313^{*}$ \\
LysoPC $(16: 0)$ & - & - & - & $-0.305^{*}$ & - \\
LysoPC(16:1) & - & $0.313^{*}$ & - & - & - \\
PC(32:1) & $0.436 * * *$ & - & - & - & - \\
PC(36:4) & - & $0.313^{*}$ & $0.331^{* *}$ & - & $-0.490^{* * *}$ \\
PC(38:4) & - & - & $0.346 * *$ & - & - \\
Hexanoylcarnitine & $-0.399 * *$ & - & - & $0.440 * * *$ & - \\
\hline
\end{tabular}

Data are presented as R values; ${ }^{*} p<0.05, * * p<0.01, * * * p<0.001 ;$ TAG- triacylglycerols, TNF- $\alpha$ - tumor necrosis factor- $\alpha$ 